\title{
A Preliminary Poetics for Interactive Drama and Games
}

\author{
Michael Mateas
}

\section{INTRODUCTION}

Interactive drama has been discussed for a number of years as a new AI-based interactive experience (Laurel 1986, Bates 1992). While there has been substantial technical progress in building believable agents (Bates, Loyall and Reilly 1992, Blumberg 1996, Hayes-Roth, van Gent and Huber 1996), and some technical progress in interactive plot (Weyhrauch 1997), no work has yet been completed which combines plot and character into a full-fledged dramatic experience. The game industry has been producing plotbased interactive experiences (adventure games) since the beginning of the industry, but only a few of them (such as The Last Express) begin to approach the status of interactive drama. Part of the difficulty in achieving interactive drama is due to the lack of a theoretical framework guiding the exploration of the technological and design issues surrounding interactive drama. This paper proposes a theory of interactive drama based on Aristotle's dramatic theory (Aristotle 330 BC) but modified to address the interactivity added by player agency. This theory both provides design guidance for interactive dramatic experiences which attempt to maximize player agency (answering the question "What should I build?") and technical direction for the AI work necessary to build the system (answering the question "How should I build it?"). In addition to clarifying notions of interactive drama, the model developed in this paper also provides general framework for analyzing player agency in any interactive experience (e.g. interactive games).

This neo-Aristotelian theory integrates Murray's (Murray 1998) proposed aesthetic categories for interactive stories and Aristotle's (Aristotle 300 B.C.) structural categories for drama. The theory borrows from Laurel's treatment of Aristotle in an interactive context (Laurel 1986, Laurel 1991) but extends it by situating Murray's category of agency within the model; the new model provides specific design guidelines for maximizing user agency. First I will give the definition of interactive drama motivating this theory and situate this definition with respect to other notions of interactive story. Next, I will present Murray's three categories of immersion, agency and transformation. Then I will present a model of Aristotle's categories relating them in terms of formal and material causation. Within this model, agency will be situated as two new causal chains inserted at the level of character. Finally, I will use the resulting model to clarify conceptual and technical issues involved in building interactive dramatic worlds, and briefly describe a current project informed by this model.

\section{DEFINING INTERACTIVE DRAMA}

Many game designers, writers and theorists have wrestled with the vexing question "what is interactive story?". This paper continues a specific thread of discussion with respect to this question, the thread begun by Laurel's adoption of an Aristotelian framework first for interactive drama (Laurel 1986) and then more generally for interactive experiences (Laurel 1991) and continued by Murray's description of the experiential pleasures and properties of interactive stories (Murray 1998). While Murray explores a variety of interactive story types, this paper will focus explicitly on the notion of interactive drama as defined in Laurel's thesis (Laurel 1986) and pursued by the Oz Project at Carnegie Mellon University (Bates, Loyall and Reilly 1992, Weyhrauch 1997).

In this conception of interactive drama, the player assumes the role of a first person character in a dramatic story. The player does not sit above the story, watching it as in a simulation, but is immersed in the story.

Following Laurel, dramatic (Aristotelian) stories are distinguished from narrative stories by the following properties:

- Enactment vs. Description

- Intensification vs. Extensification

- Unity of Action vs. Episodic Structure

Enactment refers to action. Dramas utilize action rather than description to tell a story. Intensification is achieved by arranging incidents so as to intensify emotion and condense time. In contrast, narrative forms often "explode" incidents by offering many interpretations of the same incident, examining the incident from multiple perspectives, and expanding time. Unity of action refers to the arrangement of incidents such 
that they are all causally related to a central action. One central theme organizes all the incidents that occur in the story. Narratives tend to employ episodic structure, in which the story consists of a collection of causally unrelated incidents.

Certainly not all interactive story experiences must have the properties of Aristotelian drama. In fact, most interactive story experiences built to date have either been highly episodic (generally those narrative experiences built by the game industry, e.g. adventure games), have employed a hypertextual logic of association rather than a logic of dramatic probability and causality (generally those experiences built by fine artists and writers), or have focused on story not as a highly structured experience created by an author for consumption by an audience, but rather as a shared social construction facilitating human communication (e.g. multi-user worlds such as MUDs, MOOs, and avatar spaces, massive multi-player games such as Everquest and Ultima Online, and games such as Purple Moon's Rocket series or Will Wright's The Sims). Additionally, the interaction in an interactive story does not necessarily have to be first person interaction as a character within the story. The neo-Aristotelian poetics developed here informs a specific niche within the space of interactive narrative and provides a principled way of distinguishing this niche from other interactive narrative experiences.

\section{Murray's Aesthetic Categories}

Murray (Murray 1998) proposes three aesthetic categories for the analysis of interactive story experiences: immersion, agency, and transformation.

Immersion is the feeling of being present in another place and engaged in the action therein. Immersion is related to Colridge's "willing suspension of disbelief" - when a participant is immersed in an experience, they are willing to accept the internal logic of the experience, even though this logic deviates from the logic of the real world. A species of immersion is telepresence, the feeling of being physically present (from a first person point of view) in a remote environment.

Agency is the feeling of empowerment that comes from being able to take actions in the world whose effects relate to the player's intention. This is not mere interface activity. If there are many buttons and knobs for the player to twiddle, but all this twiddling has little effect on the experience, there is no agency. Furthermore, the effect must relate to the player intention. If, in manipulating the interface elements, the player does have an effect on the world, but they are not the effects that the player intended (perhaps the player was randomly trying things because they didn't know what to do, or perhaps the player thought that an action would have one effect, but it instead had another), then there is no agency.

Transformation is the most problematic of Murray's three categories. Transformation has at least three distinct meanings.

- Transformation as masquerade. The game experience allows the player to transform themselves into someone else for the duration of the experience.

- Transformation as variety. The game experience offers a multitude of variations on a theme. The player is able to exhaustively explore these variations and thus gain an understanding of the theme.

- Personal transformation. The game experience takes the player on a journey of personal transformation. Transformation as masquerade and variety can be seen as means to effect personal transformation.

\section{InTEgrating AgenCY INTO ARISTOTLE}

Murray's categories are phenomenological categories of the interactive story experience, that is, categories describing what it feels like to participate in an interactive story. Aristotle's categories (described below) are structural categories for the analysis of drama, that is, categories describing what parts a dramatic story is made out of. The trick in developing a theoretical framework for interactive drama is integrating the phenomenological (that is, what it feels like) aspect of first person experiences with the structural aspects of carefully crafted stories. In attempting this integration, I will first discuss the primacy of the category of agency. Second, I will briefly present an interpretation of the Aristotelian categories in terms of material and formal cause. Finally, agency will be integrated into this model.

\section{Primacy of Agency}


From an interactive dramatic perspective, agency is the most fundamental of Murray's three categories. Immersion, in the form of engagement, is already implied in the Aristotelian model. Engagement and identification with the protagonist are necessary in order for an audience to experience catharsis. Transformation, in the form of change in the protagonist, also already exists in the Aristotelian model. Murray's discussion of transformation as variety, particularly in the form of the kaleidoscopic narrative that refuses closure, is contrary to the Aristotelian ideals of unity and intensification. To the extent that we want a model of interactive drama, as opposed to interactive narrative, much of Murray's discussion of transformation falls outside the scope of such a model. While immersion and transformation exist in some form in non-interactive drama, the audience's sense of having agency within the story is a genuinely new

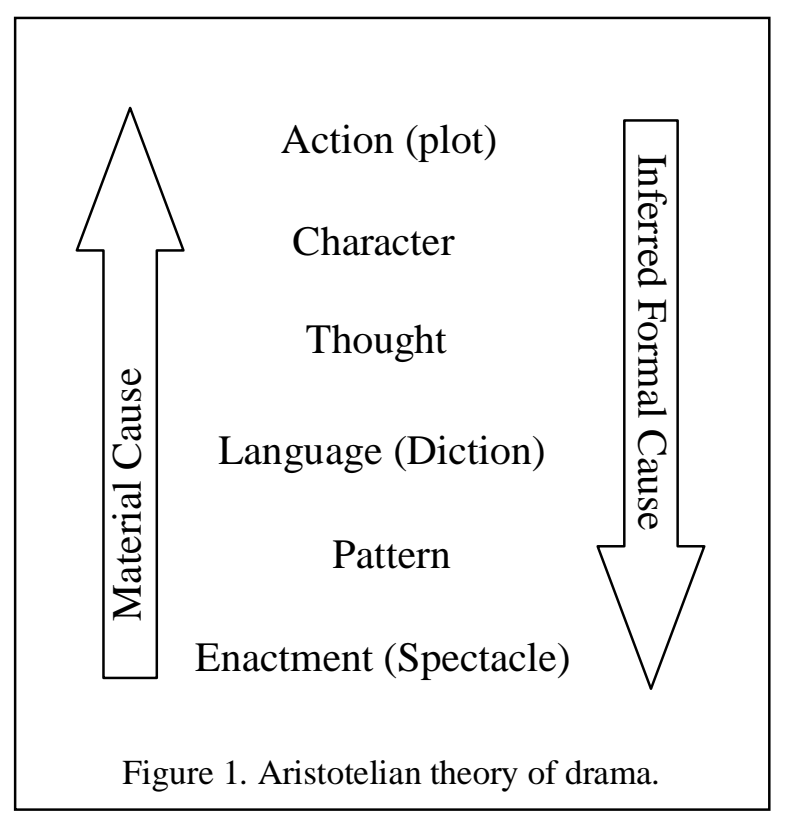
experience enabled by interactivity. For these reasons, agency will be the category integrated with Aristotle.

\section{Aristotelian Drama}

Following Laurel (Laurel 1991), Aristotle's theory of drama is represented in Figure 1. Aristotle analyzed plays in terms of six hierarchical categories, corresponding to different "parts" of a play. These categories are related via material cause and formal cause. The material cause of something is the material out of which the thing is created. For example, the material cause of a building is the building materials of which it is constructed. The formal cause of something is the abstract plan, goal or ideal towards which something is heading. For example, the formal cause of a building is the architectural blueprints.

In drama, the formal cause is the authorial view of the play. The author has constructed a plot that attempts to explicate some theme. The characters required in the play are determined by the plot; the plot is the formal cause of the characters. The character's thought processes are determined by the kind of character they are. The language spoken by the characters is determined by their thought. The patterns (song) present in the play are determined, to a large extent, by the character's language (more generally, their actions). The spectacle, the sensory display presented to the audience, is determined by the patterns enacted by the characters.

In drama, the material cause is the audience view of the play. The audience experiences a spectacle, a sensory display. In this display, the audience detects patterns. These patterns are understood as character actions (including language). Based on the character's actions and spoken utterances, the audience infers the character's thought processes. Based on this understanding of the character's thought processes, the audience develops an understanding of the characters, the character's traits and propensities. Based on all this information, the audience understands the plot structure and the theme. In a successful play, the audience is then able to recapitulate the chain of formal causation. When the plot is understood, there should be an "ah-ha" experience in which the audience is now able to understand how the characters relate to the plot (and why they must be the characters they are), why those type of characters think they way do, why they took the actions they did and said what they did, how their speech and actions created patterns of activity, and how those patterns of activity resulted in the spectacle that the audience saw. By a process of interpretation, the audience works up the chain of material cause in order to recapitulate the chain of formal cause.

\section{Interactive Drama}

Adding interaction to the Aristotelian model can be considered the addition of two new causal chains at the level of character. 
In Figure 2, the gray arrows are the traditional chains of material and formal causation. The player has

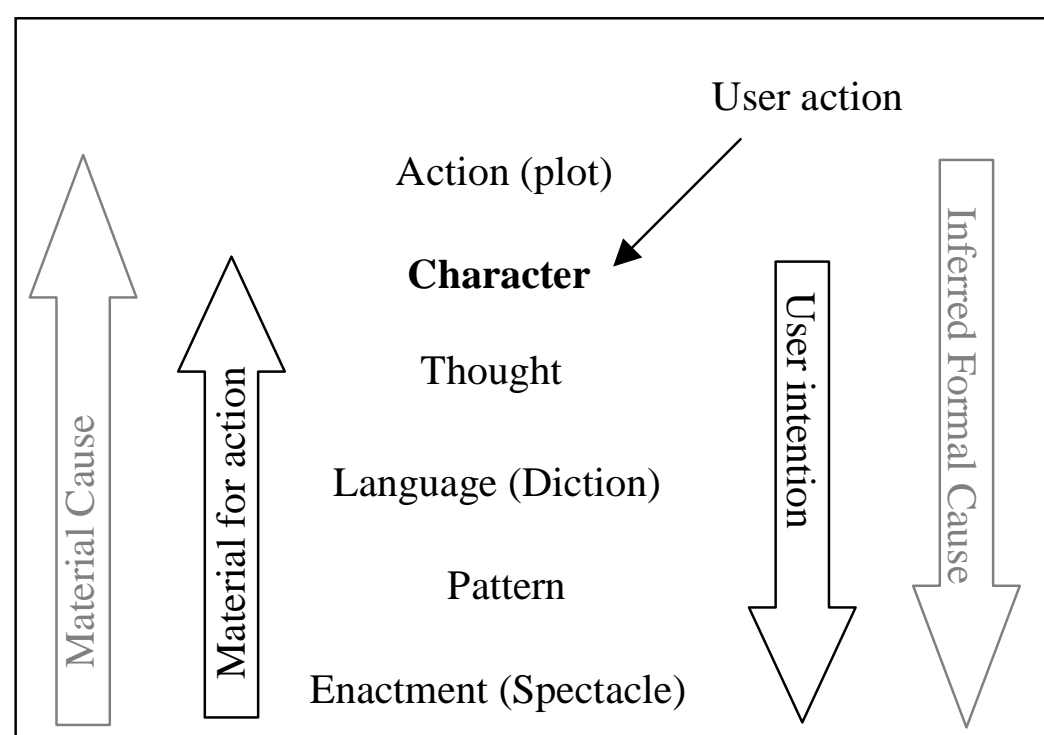

Figure 2. Neo-Aristotelian theory of drama. been added to the model as a character who can choose his or her own actions. This has the consequence of introducing two new causal chains. The player's intentions become a new source of formal causation. By taking action in the experience, the player's intentions become the formal cause of activity happening at the levels from language down to spectacle. But this ability to take action is not completely free; it is constrained from below by material resources and from above by authorial formal causation from the level of plot.

The elements present below the level of character provide the player with the material resources (material cause) for taking action. The only actions available are the actions supported by the material resources present in the game. The notion of affordance (Norman 1988) from interface design is useful here. In interface design, affordances are the opportunities for action made available by an object or interface. But affordance is even stronger than implied by the phrase "made available"; in order for an interface to be said to afford a certain action, the interface must in some sense "cry out" for the action to be taken. There should be a naturalness to the afforded action that makes it the obvious thing to do. For example, the handle on a teapot affords picking up the teapot with your hand. The handle cries out to be grasped. In a similar manner, the material resources in an interactive drama afford action. Thus these resources not only limit what actions can be taken (the negative form of constraint) but cry out to make certain actions obvious (the positive form of constraint). Several examples of the material affordances in interactive drama are provided below.

The characters in an interactive drama should be rich enough that the player can infer a consistent model of the characters' thought. If the characters' thought can be understood (e.g. goals, motivations, desires), then this thought becomes a material resource for player action. By reasoning about the other characters' thoughts, the player can take actions to influence these characters, either to change their thoughts, or actively help or hinder them in their goals and plans.

The dialog (language) spoken by the characters and the opportunities for the player to engage in dialog are another material resource for action. Dialog is a powerful means for characters to express their thoughts, thus instrumental for helping the player to infer a model of the characters' thoughts. Conversely, dialog is a powerful means to influence character behavior. If the experience makes dialog available to the player (and most contemporary interactive experiences do not), this becomes a powerful resource for expressing player intention.

The objects available in the experience (I place the presence of interactive objects somewhere between spectacle and pattern) are yet another resource for player action.

Finally, the mechanics of interaction (spectacle) provide the low-level resources for player actions. The mechanics provide the interface conventions for taking action.

In addition to the material affordances (constraints) from below, the player experiences formal constraints from above. Of course, these constraints are not directly perceived by the player, but, just as in non-interactive drama, are understood by recapitulating the author's chain of formal causation by making inferences along the chain of material causation. In non-interactive drama, understanding the formal chain of causation allows the audience to appreciate how all the action of the play stems from the dramatic necessity of the plot and theme. In interactive drama, the understanding of the formal causation from the level of plot to character additionally helps the player to have an understanding of what to do, that is, why they should take action within the story world at all. Just as the material constraints can be considered as affording action from the levels of spectacle through thought, the formal constraints afford motivation from 
the level of plot. This motivation is conveyed as dramatic probability. By understanding what actions are dramatically probable, the player understands what actions are worth considering.

\section{Agency}

We are now ready to propose a prescriptive, structural model for agency. A player will experience agency when there is a balance between the material and formal constraints. When the actions motivated by the formal constraints (affordances) via dramatic probability in the plot are commensurate with the material constraints (affordances) made available from the levels of spectacle, pattern, language and thought, then the player will experience agency. An imbalance results in a decrease in agency. This will be made clearer by considering several examples.

Many puzzle-based adventures suffer from the imbalance of providing more material affordances than formal affordances. This results in the feeling of having many things to do (places to go, objects to fiddle with) without having any sense of why any one action would be preferable to another. For example, Zork Grand Inquisitor offers a rich world to navigate and many objects to collect and manipulate. Yet, since there is no unity of action, there is no way to relate current actions to the eventual goal of defeating the Grand Inquisitor. This leaves the player in the position of randomly wandering about trying strange juxtapositions of objects. This detracts from the sense of agency - though the player can take action, this action is often not tied to a high-level player intention. Notice that adding more material opportunities for action would not help the matter. The problem is not a lack of options of things to do, the problem is having insufficient formal constraint to decide between choices.

Quake (and its ilk) induce agency by providing a nice balance between material and formal constraints. The proto-plot establishes the following formal constraints (dramatic probabilities):

1. Everything that moves will try to kill you

2. You should try to kill everything

3. You should try to move through as many levels as possible.

From these three principles, all the rest of the action follows. The material affordances perfectly balance these formal affordances. The player can run swiftly and smoothly through the space. The player can pick up a wide array of lethal weapons. The player can fire these weapons at monsters and produce satisfying, gory deaths. The monsters' behavior is completely consistent with the "kill or be killed" ethos. Everything that one would want to try and do given the formal constraints is doable. There are no extraneous actions available (for example, being able to strike up a conversation with a monster) that are not dictated by the formal constraints.

Note that though these example games are not specifically interactive drama, the model can still be used to analyze player agency within these games. Though the model is motivated by interactive drama, it can be used to analyze the sense of agency in any interactive experience by analyzing the experience in terms of the dramatic categories offered by the model. For example, though Quake has neither plot nor characters in the strict sense, there are top-down player expectations established by a "proto-plot." This "proto-plot" is communicated by the general design of the spectacle (e.g. the design of the creepy industrial mazes) as well as the actions of the characters, even if these characters do have primitive diction and thought.

In order to invoke a sense of agency, an interactive experience must strike a balance between the material and formal constraints. An experience that successfully invokes a sense of agency inhabits a "sweet spot" in design space. Trying to add additional formal constraints (more plot) or additional material constraints (more actions) to a balanced experience are likely to move it out of the sweet spot.

\section{RELATIONSHIP TO IMMERSION AND TRANSFORMATION}

Agency was taken as the fundamental Murray category to integrate with Aristotle. In this section, I examine what the new, integrated model has to say about immersion and transformation.

\section{Immersion}

Murray suggests three ways of inducing immersion: structuring participation with a mask (an avatar), structuring participation as a visit, and making the interaction conventions (the interface mechanics) seamless. These three mechanisms can be viewed in turn as a way to provide material and formal constraints, as a design suggestion for balancing the constraints, or as a design suggestion for providing effective material constraints at the level of spectacle. Agency is a necessary condition for immersion.

An avatar can provide both material and formal constraints on a player's actions. The avatar can provide character exposition through such traits as physical mannerisms and speech patterns. This character 
exposition helps the player to recapitulate the formal, plot constraints. Through both input and output filtering (e.g. the characters in Everquest, or Mateas 1997), the avatar can provide material constraints (affordances) for action.

A visit is one metaphor for balancing material and formal constraints when the material opportunities for action are limited. From the formal side, the conventions of a visit tell the player that they won't be able to do much. Visits are about just looking around, possibly being guided through a space. Given the limited expectations for action communicated by the formal constraints, the designer can get away with (and in fact, must only) provide limited material means for action.

The mechanics provide the material resources for action at the level of spectacle (the interface can be considered part of the spectacle). Providing a clean, transparent interface insures that agency (and thus immersion) will not be disrupted.

\section{Transformation}

Most of Murray's discussion of transformation examines transformation as variety, particularly in the form of kaleidoscopic narratives which can be reentered multiple times so as to experience different aspects of the story. Agency, however, requires that a plot structure be present to provide formal constraints. An openended story without a clear point of view may disrupt the plot structure too much, thus disrupting agency. However, transformation as variety is necessary to make interaction really matter. If, every time a player enters the dramatic world, roughly the same story events occur regardless of the actions taken by the player, the player's interaction would seem inconsequential; the player would actually have no real effect on the story.

One way to resolve the apparent conflict between transformation and agency is to note that agency is a first-person experience induced by making moment-by-moment decisions within a balanced (materially and formally) interactive system, while transformation as variety is a third-person experience induced by observing and reflecting on a number of interactive experiences. Imagine an interactive drama system which guides the player through a fixed plot. As the player interacts in the world, the system, through a number of clever and subtle devices, moves the fixed plot forward. Given that these devices are clever and subtle, the player never experiences them as coercive; the player is fully engaged in the story, forming intentions, acting on them, and experiencing agency. Imagine an observer who watches many players interact with this system. The observer notices that no matter what the players do, the same plot happens (meaning that roughly the same story events occur in the same order, leading to the same climax). By watching many players interact with the system, the observer has begun to discern the devices which control the plot in the face of player interaction. This observer will conclude that the player has no true agency, that the player is not able to form any intentions within the dramatic world which actually matter. But the first-time player within the world is experiencing agency. The designer of the dramatic world could conclude that since they are designing the world for the player, not for the observer, that as long as the player experiences a true sense of interactive freedom, that is, agency, transformation as variety is not an important design consideration.

The problem with this solution to the agency vs. transformation dilemma becomes apparent as the player interacts with the world a second time. On subsequent replays of the world, the player and the observer become the same person. The total interactive experience consists of both first-person engagement within the dramatic world and third-person reflection across multiple experiences in the world. In order to support the total experience, the dramatic world must support both first-person engagement and third-person reflection; must provide agency and transformation as variety.

A dramatic world supporting this total experience could provide agency (and the concomitant need to have a plot structure providing formal constraints) and transformation by actively structuring the player experience such that each run-through of the story has a clean, unitary plot structure, but multiple runthroughs have different, unitary plot structures. Small changes in the player's choices early on result in experiencing a different unfolding plot. The trick is to design the experience such that, once the end occurs, any particular run-through has the force of dramatic necessity. The story should have the dramatic probabilities smoothly narrowing to a necessary end. Early choices may result in different necessary ends later choices can have less effect on changing the whole story, since the set of dramatically probable events has already significantly narrowed. Change in the plot should not be traceable to distinct branch points; the player will not be offered an occasional small number of obvious choices that force the plot in a different direction. Rather, the plot should be smoothly mutable, varying in response to some global state which is itself a function of the many small actions performed by the player throughout the experience. 


\section{THE TYPE OF EXPERIENCE INFORMED BY THE MODEL}

This neo-Aristotelian poetics clarifies a specific conceptual experiment in the space of interactive stories. Specifically, the experiment consists of creating an interactive dramatic experience with the experiential properties of traditional drama, namely enactment, intensity, catharsis, unity and closure. The Aristotelian analytic categories describe the structure (parts and relationships) of a story experience which induces these experiential properties. The way in which interaction has been incorporated into this model clarifies what is meant by interactive dramatic experience. Here, interaction means first-person interaction as a character within the story. Further, the essential experiential property of interactivity is taken to be agency. The interactive dramatic experience should be structured in such a way as to maximize the player's sense of agency within the story. The model provides prescriptive structural guidance for maximizing agency, namely, to balance material and formal constraints. So the conceptual experiment informed by this model can be more precisely stated as follows: build a first-person, interactive dramatic world which, in addition to the classical experiential properties of Aristotelian drama, also provides the player with a strong sense of agency.

\section{TeChnical Agenda}

In addition to clarifying conceptual and design issues in interactive drama, the neo-Aristotelian model informs a technical agenda of AI research necessary to enable this kind of experience.

The primary heuristic offered by the model is that to maintain a sense of player agency in an interactive experience, material and formal constraints must be balanced. As the sophistication of the theme and plot of an experience increases, maintaining this balance will require characters whose motivations and desires are inferable from their actions. In addition, these characters will have to respond to the player's actions. Believable agents, that is, computer controlled characters with rich personality and emotion, will be necessary to provide these characters. Additionally for many plots (e.g. domestic dramas in which the plot centers around relationships, trust, betrayal, infidelity, and self-deception), language is necessary to communicate the plot. In order to convey the formal constraints provided by the plot, the characters must have a rich repertoire of dialog available. In addition, the player must be able to talk back. One can imagine a system in which the characters can engage in complex dialog but the player can only select actions from menus or click on hotspots on the screen; this is in fact the strategy employed by character-based multimedia artwork and contemporary adventure games. But this strategy diminishes agency precisely by unbalancing material and formal constraints. The characters are able to express complex thoughts through language. However, the player is not able to influence their thought except at the coarse level provided by the mouse-click interactivity. Thus maximizing player agency requires providing at least a limited form of natural language dialog.

The function of interactive characters is primarily to communicate material and formal constraints. That is, the player should be able to understand why characters take the actions they do, and how these actions relate to the plot. Sengers (Sengers 1998a) provides a nice analysis of how this focus on agents as communication vs. agents as autonomous, independent entities, results in changes in agent architectures. When the focus changes from "doing the right thing" (action selection) to "doing the thing right" (action expression), the technical research agenda changes (Sengers 1998b). The neo-Aristotelian model indicates that action expression is exactly what is needed. In addition, an interactive drama system must communicate dramatic probability (likely activity given the plot) while smoothly narrowing the space of dramatic probability over time. This means that story action must be coordinated in such a way as to communicate these plot level constraints. Thus it is not enough for an individual character's actions to be "readable" by an observer. Multiple characters must be coordinate in such a way that their joint activity communicates both formal and material (plot and character level) affordances. This requires a technical solution which blurs the firm plot/character distinction usually made in AI architectures for interactive drama (Weyhrauch 1997; Blumberg and Galyean 1995).

\section{FACADE: An INTERACTIVE DRAMA GUIDED BY THE MODEL}

The author is currently engaged in a three year collaboration to build Facade (Mateas \& Stern, 2000), an interactive story world that seeks to carry out the conceptual and technical experiment informed by the neoAristotelian poetics. Together we will: 
- create a compelling, well-written story that obeys dramatic principles, designed with many potential ways to play out;

- build artificial intelligence (AI) that can control the behavior of realtime-animated computer characters, to be used for performing the roles of all but one of the characters in the story

- create a user interface that allows the player to easily move within the world, and converse and gesture with the computer characters;

- $\quad$ build AI that can understand a natural language and gestural input within the context of the story;

- build AI that can integrate the user's interactions into the space of potential plot directions and character behaviors in the story;

- collaborate with voice actors and animators to author spoken dialogue, character behavior and story events within the engine, to construct the finished story world.

\section{Story Requirements}

The story requirements describe the properties we wish our story to have. These are not intended to be absolute requirements, that is, this is not a description of the properties that all interactive stories must have. Rather, these requirements are the set of assumptions grounding the design of the particular interactive story we intend to build.

* Short one-act play. Any one run of the scenario should take the player 15 to 20 minutes to complete. We focus on a short story for a couple of reasons. Building an interactive story has all the difficulties of writing and producing a non-interactive story (film or play) plus all the difficulty of supporting true player agency in the story. In exploring this new interactive art form it makes sense to first work with a distilled form of the problem, exploring scenarios with the minimum structure required to support dramatically interesting interaction. In addition, a short one-act play is an extreme, contrarian response to the many hours of game play celebrated in the design of contemporary computer games. Instead of providing the player with 40 to 60 hours of episodic action and endless wandering in a huge world, we want to design an experience that provides the player with 15 to 20 minutes of emotionally intense, tightly unified, dramatic action. The story should have the intensity, economy and catharsis of traditional drama.

* Relationships. Rather than being about manipulating magical objects, fighting monsters, and rescuing princesses, the story should be about the emotional entanglements of human relationships. We are interested in interactive experiences that appeal to the adult, non-computer geek, movie-and-theater-going public.

* Three characters. The story should have three characters, two controlled by the computer and one controlled by the player. Three is the minimum number of characters needed to support complex social interaction without placing the responsibility on the player to continually move the story forward. If the player is shy or confused about interacting, the two computer controlled characters can conspire to set up dramatic situations, all the while trying to get the player involved.

* The player should be the protagonist. Ideally the player should experience the change in the protagonist as a personal journey. The player should be more than an "interactive observer," not simply poking at the two computer controlled characters to see how they change.

* Embodied interaction should matter. Though dialog should be a significant (perhaps the primary) mechanism for character interaction, it should not be the sole mechanism. Embodied interaction, such as moving from one location to another, picking up an object, or touching a character, should play a role in the action. These physical actions should carry emotional and symbolic weight, and should have a real influence on the characters and their evolving interaction. The physical representation of the characters and their environment should support action significant to the plot.

* Action takes place in a single location. This provides unity of space and forces a focus on plot and character interaction.

* The player should not be over-constrained by a role. The amount of non-interactive exposition describing the player's role should be minimal. The player should not have the feeling of playing a role, of actively having to think about how the character they are playing would react. Rather, the player should be able to be themselves as they explore the dramatic situation. Any role-related scripting of the interactor (Murray 1998) should occur as a natural by-product of their interaction in the world. The player should "ease into" their role; the role should be the "natural" way to act in the environment, given the dramatic situation.

\section{Story}

Our story, which satisfies these story requirements, is a domestic drama in which a married couple has invited the player over for dinner. (Assume for the moment that the players character is male.) Grace and 
Trip are apparently a model couple, socially and financial successful, well-liked by all. Grace and Trip both know the player from work. Trip and the player are friends; Grace and the player have gotten to know each other fairly recently. Shortly after arriving at their house for dinner, Grace confesses to the player that she has fallen in love with him. Throughout the rest of the evening, the player discovers that Grace and Trip's marriage is actually falling apart. Their marriage has been sour for years; deep differences, buried frustrations and unspoken infidelities have killed their love for each other. How the facade of their marriage cracks, what is revealed, and the final disposition of Grace and Trip's marriage, and Grace and the player's relationship, depends on the actions of the player. The story's controlling idea: To be happy you must be true to yourself.

The above story description assumes a male player. Ideally the player will be able to choose whether they wish to be a male or female player (important to support the player should not be over-constrained by a role story requirement). In the case of a female player, the story would play itself out symmetrically, with Trip confessing his love for the player. For the purposes of this story, we are assuming heterosexual relationships. Ideally, sexual orientation would be selectable by the player as well.

\section{Interface}

The story world is presented to the player as an animated, 3D environment. The environment and characters within the environment are rendered in an illustrative style reminiscent of graphic novels. The player is able to move about this environment from a first-person point-of-view, gesture and pick up objects, and converse with the other characters by typing. The computer-controlled characters look directly out of the screen to gesture and talk to the player. The conversation discourse is real-time; that is, if the player is typing, it is as if they are speaking those words in (pseudo) real-time.

\section{Story structure}

The story is structured as a classic Aristotelian plot arc. The AI plot system explicitly attempts to change dramatic values (e.g. the love between Trip and Grace, the trust between the player and Trip) in such a way as to make a well-formed plot arc happen. In the theory of (classical) dramatic writing, the smallest unit of value change is the beat (McKee 1997). Roughly, a beat consists of an action/reaction pair between characters. Beats are sequenced to make scenes, scenes to make acts, acts to make stories. The AI plot system contains a library of beats appropriate for our story. The system dynamically sequences beats in such a way as to respond to player activity and yet maintain a well-formed plot arc. For the player, each run-through of the story should have the force of dramatic necessity. Explicit decision points, which would highlight the non-linearity of the story, should not be visible. However, in multiple run-throughs of the story, the player's actions have a significant influence on what events occur in the plot, which are left out, and how the story ends. Only after playing the experience 6 or 7 times should the player begin to feel they have "exhausted" the interactive story. In fact, full appreciation of the experience requires the story be played multiple times. In Façade, our goal is to create an interactive story experience which provides the player with the agency to have an effect on the trajectory of the story, yet has the feel of a traditional, linear, dramatic experience.

\section{CONCLUSION}

In this paper, Murray's concept of agency was integrated into Laurel's Aristotelian structural model to yield a proposed Aristotelian interactive poetics. This model illuminates the general conditions under which a user will experience agency in any interactive experience and provides design and technology guidance for the particular case of building interactive dramatic experiences.

\section{BIBLIOGRAPHY}

Aristotle, 330 BC. The Poetics. Mineola, New York: Dover, 1997.

Bates, J. 1992. Virtual Reality, Art, and Entertainment. Presence: The Journal of Teleoperators and Virtual Environments 1(1): pages 133-138.

Bates, J.; Loyall, A. B.; Reilly, W. S. 1992. Integrating Reactivity, Goals and Emotion in a Broad Agent, Technical Report, CMU-CS-92-142, Department of Computer Science, Carnegie Mellon Univ.

Blumberg, B. 1996. Old Tricks, New Dogs: Ethology and Interactive Creatures. Ph.D. Dissertation. MIT 
Media Lab. 1996.

Blumberg, B. and Galyean, T. 1995. Multi-level Direction of Autonomous Creatures for Real-Time Virtual Environments. In Proceedings of SIGGRAPH 95.

Hayes-Roth, B., van Gent, R. and Huber, D. 1996. Acting in character. In R. Trappl and P. Petta (Eds.), Creating Personalities for Synthetic Actors. Also available as Stanford Knowledge Systems Laboratory Report KSL-96-13, 1996.

Laurel, Brenda. 1991. Computers as Theatre. Reading, MA: Addison-Wesley, 1991.

Laurel, Brenda. 1986. Towards the Design of a Computer-Based Interactive Fantasy System. Ph.D. Diss., The Ohio State University, 1986.

Mateas, M. \& Stern, A. 2000. Towards Integrating Plot and Character for Interactive Drama. Working notes of the Socially Intelligent Agents: Human in the Loop Symposium, 2000 AAAI Fall Symposium Series. Menlo Park, CA.: AAAI Press.

Mateas, M. 1997. Computational Subjectivity in Virtual World Avatars. Working notes of the Socially Intelligent Agents Symposium, 1997 AAAI Fall Symposium Series. Menlo Park, Calif.: AAAI Press.

McKee, R. 1997. Story: Substance, Structure, Style and the Principles of Screenwriting. New York, NY: HarperCollins.

Murray, Janet. 1998. Hamlet on the Holodeck. Cambridge, MA: MIT Press, 1998.

Norman, Don. 1988. The Design of Everyday Things. New York, NY: Doubleday, 1988.

Sengers, P. 1998a. Anti-Boxology: Agent Design in Cultural Context. Ph.D. Thesis, School of Computer Science, Carnegie Mellon University. Technical Report CMU-CS-98-151. 1998.

Sengers, P. 1998b. Do the Thing Right: An Architecture for Action Expression. Proceedings of the Second International Conference on Autonomous Agents, May 1998, pp. 24-31.

Weyhrauch, P. 1997. Guiding Interactive Drama. Ph.D. Thesis, School of Computer Science, Carnegie Mellon University. Technical Report CMU-CS-97-109. Pittsburgh, PA: 1997. 